\title{
Advanced glycation end products derived from serum albumin modification by glucose (AGE-1) reflect clustering of lipid-associated metabolic abnormalities and are decreased in patients treated with acarbose: A cross-sectional study
}

\author{
Agnieszka Bronowicka-Szydełko ${ }^{1, A, D}$, Małgorzata Krzystek-Korpacka, ${ }^{1, C, D}$, Aleksandra Kuzann ${ }^{1, C}$, Kinga Gostomska-Pampuch ${ }^{1, C}$, \\ Małgorzata Gacka ${ }^{2, B}$, Urszula Jakobsche-Policht ${ }^{2, B}$, Rajmund Adamiec ${ }^{2, D, E}$, Andrzej Gamian ${ }^{1,3, E, F}$ \\ ${ }^{1}$ Department of Medical Biochemistry, Wroclaw Medical University, Poland \\ ${ }^{2}$ Department of Angiology, Diabetes and Hypertension, Wroclaw Medical University, Poland \\ ${ }^{3}$ Laboratory of Medical Microbiology, Hirszfeld Institute of Immunology and Experimental Therapy, Polish Academy of Sciences, Wrocław, Poland \\ A - research concept and design; B - collection and/or assembly of data; $\mathrm{C}$ - data analysis and interpretation; \\ $\mathrm{D}$ - writing the article; $\mathrm{E}$ - critical revision of the article; $\mathrm{F}$ - final approval of the article
}

Address for correspondence

Agnieszka Bronowicka-Szydełko

E-mail: agnieszka.bronowicka-szydelko@umed.wroc.pl

Funding sources

This work was supported by National Science Centre; grant No. 2012/05/N/NZ5/00836 (A. Bronowicka-Szydełko).

Conflict of interest

None declared

Received on November 7, 2018

Review on December 12, 2018

Accepted on September 25, 2019

Published online on March 24, 2020

Cite as

Bronowicka-Szydełko A, Krzystek-Korpacka M, Kuzan A, et al. Advanced glycation end products derived from serum albumin modification by glucose (AGE-1) reflect clustering of lipid-associated metabolic abnormalities and are decreased in patients treated with acarbose: $\mathrm{A}$ cross-sectional study. Adv Clin Exp Med. 2020;29(3):275-284.

doi:10.17219/acem/112611

DOI

10.17219/acem/112611

Copyright

Copyright by Author(s)

This is an article distributed under the terms of the

Creative Commons Attribution 3.0 Unported (CC BY 3.0)

(https://creativecommons.org/licenses/by/3.0/)

\begin{abstract}
Background. Advanced glycation end products (AGES) are formed during protein modification by a reduction of sugars or reactive aldehydes. Depending on the pathology, various AGEs may be formed. They are stable compounds and are considered as potential diseases markers.

Objectives. The objective of this study was to assess glucose-mediated albumin modification that yields non-standard epitopes of AGES (AGE-1) in diabetes and in associated metabolic abnormalities.

Material and methods. The AGE-1, expressed as median AGE-1 level and AGE-1 positivity, was determined in 246 individuals (198 with prediabetes/diabetes) using a new slot-dot-blot method (allowing for detection of barely traceable analytes) and related to the presence of diabetes-associated metabolic abnormalities and complications, and treatment.
\end{abstract}

Results. The AGE-1 level was higher in patients with prediabetes/diabetes than in controls. Its elevation was associated with metabolic syndrome (MetS), obesity, hyperlipidemia, and non-alcoholic fatty liver disease (NAFLD) but not with diabetic control or micro- and macroangiopathy, except for atherosclerotic plaques formation in carotid arteries. The AGE-1-positive patients had higher triglycerides and lower high-density lipoprotein (HDL)-cholesterol. In patients untreated with aspirin, AGE-1 positivity was associated with higher C-reactive protein (CRP) level. Treatment with aspirin, sulfonylureas and gliptins was associated with higher AGE-1 level and with dyslipidemia medications with higher AGE-1 positivity. In patients with abnormal glucose metabolism, acarbose treatment was associated with lower AGE-1 positivity. Multivariate analysis showed MetS, carotid artery plaques, NAFLD, and treatment with aspirin and acarbose to be independently associated with AGE-1 positivity.

Conclusions. Unlike standard AGEs, AGE-1 is more tightly associated with abnormalities in lipid than glucose metabolism, and lower in patients treated with acarbose but not with other antidiabetics.

Key words: metabolic syndrome, diabetes, atherosclerosis, acarbose, advanced glycation end products 


\section{Introduction}

Diabetes is increasingly viewed as a spectrum of disorders, such as prediabetes, type 1 (T1DM) and type 2 (T2DM) diabetes, gestational and neonatal diabetes, maturity onset diabetes in young people, and latent autoimmune diabetes in adults. ${ }^{1}$ The common denominator of these disorders is abnormal glucose level or tolerance, accompanied by lack of or inadequate response to insulin. With the current estimates of $8.5 \%$ of adult population worldwide being affected, the prevalence of diabetes is still on the rise and parallels the increasing prevalence of overweight/obesity. Diabetes is one of the top causes of mortality for both women and men. If not controlled, it leads to complications, such as cardio- and cerebrovascular disease, further increasing the risk of premature death. ${ }^{2}$ Obesity and abnormal glucose constitute the core features of metabolic syndrome (MetS). The remaining components include atherogenic dyslipidemia and hypertension. Metabolic syndrome is viewed as a cluster of metabolic risk factors for diabetes and cardiovascular disease with the risk prediction increasing with the number of co-existing metabolic abnormalities. ${ }^{3}$

Glycation is a non-enzymatic reaction occurring between reducing carbohydrates or reactive aldehydes and amino groups of macromolecules. The accumulation of reaction products, that is, advanced glycation end products (AGEs), is particularly evident during aging. However, AGEs formation is accelerated in diabetes, facilitated by disease-associated hyperglycemia, oxidative stress and low-grade inflammation. ${ }^{4}$

Enhanced accumulation of AGEs and resulting modifications of macromolecules contribute to cardio- and cerebrovascular complications. ${ }^{5}$ Accordingly, intervention studies have shown that low-AGE diet improves insulin sensitivity and thus may reduce diabetes and cardiovascular disease risks. ${ }^{6}$ Advanced glycation end products, in turn, further exacerbate oxidative stress and inflammation. Additionally, AGEs form covalent crosslinks with extracellular matrix proteins, contributing to cardiac fibrosis, stiffening of the arteries and abnormal vasodilator response to nitric oxide. ${ }^{5}$ Moreover, glycoxidation-caused alterations in the protein conformation may lead to the formation of neo-epitopes and the rise of autoantibodies, causing adverse immunological responses. ${ }^{7}$

The causative role for AGEs in the pathogenesis and progression of metabolic diseases is widely accepted but to what degree particular AGEs are harmful and thus clinically relevant, needs to be elucidated. Advanced glycation end products are an extremely diverse group of compounds displaying distinct physicochemical and immunogenic properties. $^{8}$ The type of AGE formed depends not only on the modified macromolecule and glycating agent but also on the reaction conditions. Despite their enormous variety, only the structures of epitopes of several AGEs have been identified thus far. ${ }^{9}$ Consequently, quantification of AGEs and studies on their association with disease pathology either concern the so-called "total AGEs" or are focused on a few specific AGEs, such as $\mathrm{N}^{\varepsilon}$-carboxymethyllysine (CML), $\mathrm{N}^{\varepsilon}$-carboxyethyllysine (CEL) or imidazolones. The contribution of other AGEs remains largely unknown. ${ }^{10}$ Moreover, in easily accessible biological material, such as blood or urine, specific AGE epitopes are present in trace amounts and are consequently difficult to detect and quantify. Sophisticated laboratory techniques that are required to assess the majority of specific AGE epitopes ${ }^{11}$ reduce the applicability of AGEs as biomarkers in clinical practice. In turn, available immunoassays mostly use polyclonal antibodies and thus are not suitable for detection of individual AGEs. Moreover, CML modified proteins appear to be the main epitope for anti-AGE antibodies. ${ }^{12}$ Yet, CML-modification has been shown to better reflect lipid peroxidation than glycation. ${ }^{13}$ Therefore, the need to use the antibodies against non-CML AGEs in studies of the effects of glycation has been emphasized. ${ }^{14}$

This study was designed to assess, using a newly developed immunoassay based on monoclonal, commercially available antibodies, the glycation of serum albumin by glucose with the formation of epitopes other than typical ones (AGE-1) in diabetes and associated metabolic abnormalities, with reference to treatment.

\section{Material and methods}

\section{Patients}

The study population consisted of 246 individuals: 198 with and 48 without abnormalities in glucose metabolism. Patients with deregulated glucose metabolism were recruited from the Department of Angiology, Diabetes and Hypertension of Wroclaw Medical University, Poland. The group consisted of individuals with prediabetes $(n=10)$ or with diabetes mellitus ( $\mathrm{n}=188$; 14 with T1DM, 163 with T2DM and 11 with a secondary diabetes (T3DM) - the main cause of secondary diabetes was pancreatitis).

Table 1. Characteristics of study population

\begin{tabular}{|l|c|c|c|}
\hline \multicolumn{1}{|c|}{ Variables } & Controls & $\begin{array}{c}\text { Patients with } \\
\text { abnormal glucose } \\
\text { metabolism }\end{array}$ & p-value \\
\hline Number of cases & 48 & 198 & - \\
\hline $\begin{array}{l}\text { Sex (F/M), } n \\
\text { Age [years], } \\
\text { mean (range) }\end{array}$ & $26 / 22$ & $121 / 77$ & $0.414^{\mathrm{F}}$ \\
\hline $\begin{array}{l}\text { AGE-1 positivity, } \mathrm{n}(\%) \\
(59.7-63.2)\end{array}$ & $17(35.4)$ & $63.5(61-65)$ & $0.249^{\mathrm{M}}$ \\
\hline $\begin{array}{l}\text { AGE-1 [AU], } \\
\text { mean (range) }\end{array}$ & $0(0-572)$ & $4,888(0-13,061)$ & $0.004^{\mathrm{M}}$ \\
\hline
\end{tabular}

Continuous data presented as medians accompanied with 95\% confidence interval $(95 \% \mathrm{Cl})$. $^{\mathrm{F}}$ - Fisher's exact test; ${ }^{\mathrm{M}}$ - Mann-Whitney U test; F/M - female-to-male ratio; AGE - advanced glycation end products. AGE-1-positive samples were defined as samples with measurable AGE-1 (AGE-1 > 0); $n$ - number of observation; $A U$ - arbitrary units. 
The control group, without known abnormalities in glucose metabolism, consisted of patients with atherosclerosis $(\mathrm{n}=18)$ and apparently healthy blood donors $(\mathrm{n}=30)$, recruited from the Regional Center of Blood Donation and Therapy in Wrocław. The inclusion criteria were the following: age $>50$ years, no known systemic disease, dementia, depression or ongoing inflammation, and fasting glucose $<100 \mathrm{mg} / \mathrm{dL}$.

Demographic, clinical and laboratory data was collected prospectively. The characteristics of the study population are given in Table 1, while data on treatment and the coexistence of other abnormalities is presented in Tables 2-5.

\section{Definitions}

Prediabetes was defined as impaired fasting plasma glucose (100-125 mg/dL) or glucose tolerance (2-hour plasma glucose in the 75-gram oral glucose tolerance test $=140$ $199 \mathrm{mg} / \mathrm{dL}$ ). ${ }^{2}$ The World Health Organization (WHO) criteria for diabetes were applied. Diabetes was considered controlled if the percentage of glycation of the hemoglobin $\mathrm{A}_{1} \mathrm{c}$ chain $\left(\mathrm{HbA}_{1} \mathrm{c}\right)$ was $\leq 6.4 \% .^{2}$

The WHO classification of adult weight according to body mass index (BMI) was applied with BMI $25-30 \mathrm{~kg} / \mathrm{m}^{2}$ indicative of overweight (pre-obese) and BMI $\geq 30 \mathrm{~kg} / \mathrm{m}^{2}$ indicative of obesity (class I: $30-35 \mathrm{~kg} / \mathrm{m}^{2}$, class II: $35-$ $40 \mathrm{~kg} / \mathrm{m}^{2}$, class III: $\left.\geq 40 \mathrm{~kg} / \mathrm{m}^{2}\right) .{ }^{15}$

Metabolic syndrome was defined according to the International Diabetes Federation (IDF) criteria ${ }^{16}$ as the coexistence of central obesity plus any of the 2 following: hypertriglyceridemia ( $\geq 150 \mathrm{mg} / \mathrm{dL}$ ) or receiving treatment $(\mathrm{RT})$, low level of high-density lipoprotein (HDL)-cholesterol $(<50 \mathrm{mg} / \mathrm{dL}$ in women and $<40 \mathrm{mg} / \mathrm{dL}$ in men), hypertension ( $\geq 130 \mathrm{~mm} \mathrm{Hg}$ or $\geq 85 \mathrm{~mm} \mathrm{Hg}$ for systolic or diastolic, respectively) or $\mathrm{RT}$, glucose $\geq 100 \mathrm{mg} / \mathrm{dL}$, or diagnosed with diabetes and/or RT.

Hyperlipidemia was defined according to the guidelines of the European Society of Cardiology (ESC) and the European Atherosclerosis Society (EAS) ${ }^{17}$ as elevated total cholesterol ( $\geq 190 \mathrm{mg} / \mathrm{dL}$ ), and/or low-density lipoprotein (LDL)-cholesterol ( $\geq 115 \mathrm{mg} / \mathrm{dL})$ and/or triglycerides ( $\geq 150 \mathrm{mg} / \mathrm{dL}$ ) or RT.

Hyperuricemia definition was based on the NHANES-III criteria ${ }^{18}$ as a serum urate level of $7.0 \mathrm{mg} / \mathrm{dL}$ in men and $5.7 \mathrm{mg} / \mathrm{dL}$ in women.

Macroangiopathy (atherosclerosis of arteries) was diagnosed in patients who had a myocardial infarction or stroke, percutaneous coronary intervention or coronary artery bypass graft, acute coronary syndrome (defined as treatment in a hospital as a consequence of 1 or more episodes of ischemic discomfort at rest and characterized by electrocardiogram changes and/or elevation of a cardiac serum marker to an extent not indicative of a myocardial infarction), objective evidence of coronary artery disease (defined as positive exercise test, angiography with at least 1 stenosis $>50$ ), symptomatic peripheral arterial obstructive disease (confirmed by an ankle/brachial pressure index $<0.90$ or an amputation), stenosis of carotid artery, or cardiovascular death.

Microangiopathy was diagnosed in patients with diabetic nephropathy (albuminuria $>30 \mathrm{mg} / \mathrm{g}$, creatinine and estimated glomerular filtration rate (eGFR) assessed using Chronic Kidney Disease Epidemiology Collaboration (CKD-EPI) creatinine equation), diabetic retinopathy (microaneurysms, dot-blot hemorrhages, flame-shaped hemorrhages, retinal edema and hard exudates, cottonwool spots, venous loops and venous beading, intraretinal microvascular abnormalities, hemorrhage into the vitreous, traction retinal detachments, macular edema etc., detected using ophthalmoscopy, fundus fluorescein angiography or optical coherence tomography), or polyneuropathy (diagnosed after excluding other possible causes with the assessment of pain sensation according to the pinprick test (absent or decreased), electromyography, and/or clinical evaluation using, e.g., Neuropathy Impairment Score).

\section{Ethical considerations}

The study protocol was approved by the Medical Ethics Committee of Wroclaw Medical University (approvals No. KB-303/2010 and No. KB-384/2012) and was in accordance with the ethical standards formulated in the Helsinki Declaration of 1975. Informed consent was obtained from all subjects.

\section{Analytical methods}

Serum samples were obtained from clotted (15 min, room temperature) and centrifuged $(15 \mathrm{~min}, 400 \times \mathrm{g})$ blood drawn using venipuncture into serum-separator tubes following a 12-hour overnight fast.

The AGE-1 level was assessed in sera using monoclonal anti-AGE-1 antibodies (clone No. 7C1; CosmoBio, Tokyo, Japan) and a slot-dot-blot method, recently developed in our laboratory (manuscript submitted). The antibodies react selectively with glucose-modified (AGE-1) bovine serum albumin (BSA) and are confirmed to not crossreact with methylglyoxal-modified BSA (AGE-4), glyceraldehyde-modified BSA (AGE-2), glycolaldehyde-modified BSA (AGE-3), glyoxal-modified BSA (AGE-5), 3-DG-imidazolone-modified BSA (AGE-6), carboxymethyllysinemodified BSA (CML), carboxyethyllysine-modified BSA (CEL), or native BSA. ${ }^{19}$ Briefly, diluted sera were applied into methanol-activated PVDF membranes in Bio-Dot ${ }^{\circledR}$ SF Microfiltration Apparatus (BioRad, Hercules, USA). Membranes were blocked with 5\% skimmed milk and incubated with primary antibodies (1:1,000), followed by secondary antibodies (goat anti-mouse IgG; 1:2,000; Jackson ImmunoResearch Europe Ltd., Cambridgeshire, UK) conjugated with horseradish peroxidase. 3-amino-9-ethylcarbazole (Sigma-Aldrich, St. Louis, USA) was used as a peroxidase 
substrate and the density of developed color, proportional to AGE-1 concentration, was determined using densitometry and expressed in arbitrary units (AU).

Data on biochemical indices, determined using standard automated procedures, were collected at the time of blood sampling for AGE-1 determination.

\section{Statistical analysis}

Data distribution was tested using KolmogorovSmirnov test. Data on AGE-1 is presented as medians with 95\% confidence interval (95\% CI) and analyzed using Kruskal-Wallis H test or Mann-Whitney U test. Advanced glycation end products distribution was compared using one-way analysis of variance (ANOVA) and highsensitive C-reactive protein (hsCRP), and $\mathrm{HbA}_{1} \mathrm{c}$ levels were analyzed using t-test for independent samples. Frequency analysis was conducted using Fisher's exact test or $\chi^{2}$ test. Correlation was analyzed using Spearman's test. Logistic regression (stepwise method) with $\mathrm{p}<0.05$ as an entrance criterion and $p>0.1$ as a removal criterion was applied to identify independent predictors of AGE-1 positivity. Odds ratios (ORs) with 95\% CI were calculated for significant variables.

All calculated $\mathrm{p}$-values were two-sided; $\mathrm{p} \leq 0.05$ was considered statistically significant. Statistical analysis was conducted using MedCalc Statistical Software v. 17.9.6 (MedCalc Software bvba, Ostend, Belgium; http://www. medcalc.org; 2017).

\section{Results}

\section{AGE-1 and abnormal glucose metabolism}

Individual distribution of AGE-1 in the study population is depicted in Fig. 1. A total of 120 out of 246 study

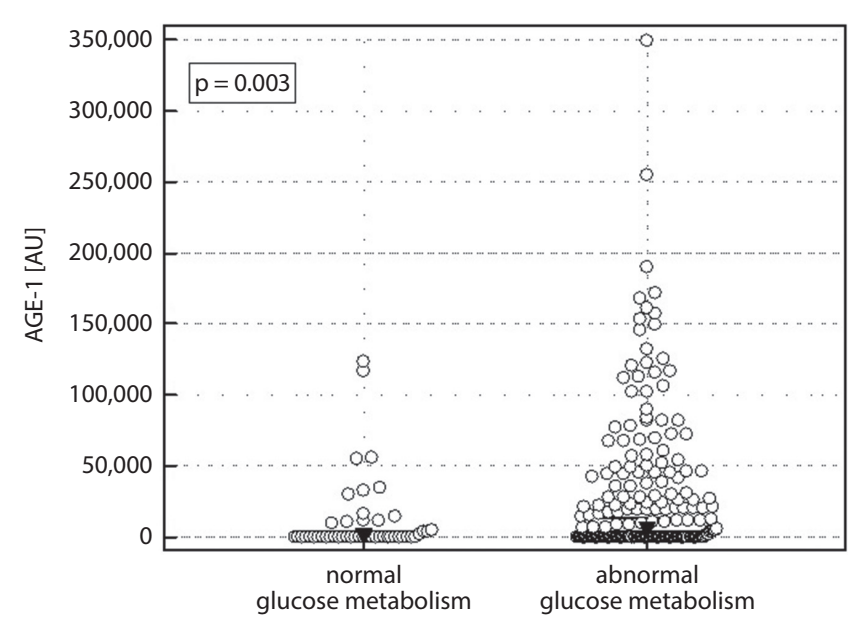

Fig. 1. AGE-1 in individuals with normal and abnormal glucose metabolism. Open circles represent individual AGE-1 levels, black triangles represent median values, whiskers represent 95\% confidence interval $(95 \% \mathrm{Cl})$ around median. Data analyzed using the Mann-Whitney $U$ test participants had undetectable AGE-1 level and there was substantial variability among the AGE-1-positive samples (range: 141-348,864 AU). Therefore, the data was analyzed by comparing both AGE-1 levels and the proportions of AGE-1-positive samples (defined as samples with measurable AGE-1 levels), referred to as AGE-1 positivity.

The AGE-1 positivity and median AGE-1 levels were significantly higher in patients with abnormal glucose metabolism than in controls (Table 1).

Detailed analysis showed that patients with prediabetes, T1DM and T2DM had comparable AGE-1 positivity, higher than T3DM patients or controls, with the difference between T2DM and controls being statistically significant. Patients with uncontrolled diabetes had higher AGE-1 level than those with controlled disease, but the difference did not reach statistical significance (Table 2).

We additionally compared AGE-1 and $\mathrm{HbA}_{1} \mathrm{c}$ levels in patients with prediabetes and diabetes (regardless of its type). The AGE-1 level in prediabetes was comparable to diabetes $(10,629 \mathrm{AU}(0-130,721 \mathrm{AU})$ vs 4,699 AU $(0-12,411 \mathrm{AU}), \mathrm{p}=0.541)$ whereas $\mathrm{HbA}_{1} \mathrm{c}$ level was significantly lower $(5.5 \mathrm{~g} / \mathrm{dL}(5.3-5.6 \mathrm{~g} / \mathrm{dL})$ vs $7.3 \mathrm{~g} / \mathrm{dL}$ (6.3-8.8 g/dL), p < 0.001). Also, AGE-1 positivity was similar between patients with prediabetes and diabetes (60\% vs $54.8 \%$, p = 0.748).

Patients treated with metformin, sulfonylureas or gliptins had higher AGE-1 level, significantly so in the case of sulfonylureas and gliptins, without a significant difference in AGE-1 positivity (Table 3 ). When the analysis was restricted to patients diagnosed with abnormal glucose metabolism, only the difference in median AGE-1 level between patients treated and untreated with gliptins remained significant (26,251 AU (0-49,783 AU) vs $2012 \mathrm{AU}$ (0-10,452 AU), $\mathrm{p}=0.028)$. In turn, AGE-1 positivity tended to be lower in acarbose-treated patients (Table 3 ). The difference gained significance when analyzed exclusively in patients with abnormal glucose metabolism $(34.8 \%$ in treated vs $57.7 \%$ in untreated, $\mathrm{p}=0.046$ ).

\section{AGE-1 and obesity}

Underweighted/normal weight patients had significantly lower AGE-1 level than patients with overweight or obesity (Table 2). There was also a clear tendency towards a difference in AGE-1 positivity, with the differences between underweight/normal weight and overweight patients significant in paired analysis, and the differences between underweight/normal weight and obese or morbidly obese patients with $\mathrm{p} \leq 0.06$.

\section{AGE-1 and dyslipidemia}

The AGE-1 level was significantly higher in patients with hyperlipidemia and in patients with non-alcoholic fatty liver disease (NAFLD; Table 2). 
Table 2. AGE and metabolic abnormalities

\begin{tabular}{|c|c|c|c|c|}
\hline Variables & $\begin{array}{l}\text { AGE-1 level [AU] } \\
\text { median }(95 \% \mathrm{CI})\end{array}$ & $p$-value ${ }^{\mathrm{M}}$ & $\begin{array}{c}\text { AGE-1 positivity } \\
n(\%)\end{array}$ & $p$-value ${ }^{F}$ \\
\hline $\begin{array}{l}\text { Type of deregulation } \\
\text { normal (controls; } n=48) \\
\text { prediabetes }(n=10) \\
\text { T1DM }(n=14) \\
\text { T2DM }(n=155) \\
\text { T3DM }(n=19)\end{array}$ & $\begin{array}{c}0(0-572)^{1} \\
10,629(0-130,721) \\
7,146(0-18,710) \\
4,945(0-18,964)^{2} \\
0(0-45,705)\end{array}$ & 0.044 & $\begin{array}{c}17(35.4)^{1} \\
6(60) \\
9(64.3) \\
90(55.2)^{2} \\
4(36.4)\end{array}$ & 0.088 \\
\hline $\begin{array}{l}\text { Diabetes control* } \\
\text { controlled }(n=52) \\
\text { uncontrolled }(n=136)\end{array}$ & $\begin{array}{c}0(0-12,096) \\
7,970(0-19,186)\end{array}$ & 0.266 & $\begin{array}{l}25(48.1) \\
78(57.4)\end{array}$ & 0.258 \\
\hline $\begin{array}{l}\text { Atherosclerosis } \\
\text { no }(n=33) \\
\text { yes }(n=183)\end{array}$ & $\begin{array}{c}0(0-13,072) \\
6,141(0-14,739)\end{array}$ & 0.355 & $\begin{array}{c}15(45.5) \\
100(54.6)\end{array}$ & 0.331 \\
\hline $\begin{array}{l}\text { Weight** } \\
\text { underweight/normal }(n=54) \\
\text { overweight }(n=71) \\
\text { obesity class I/II }(n=83) \\
\text { obesity class III }(n=8)\end{array}$ & $\begin{array}{c}0(0-1,452)^{3} \\
14,619(0-25,178) \\
4,830(0-18,856) \\
33,356(0-52,711)\end{array}$ & 0.008 & $\begin{array}{l}21(38.9) \dagger \\
42(59.2) \dagger \\
46(55.4) \\
6(75)\end{array}$ & 0.067 \\
\hline $\begin{array}{l}\text { Weight (dichotomized)** } \\
\text { underweight/normal }(n=54) \\
\text { overweight/obesity }(n=162)\end{array}$ & $\begin{array}{c}0(0-1,452) \\
10,655(144-20,232)\end{array}$ & 0.001 & $\begin{array}{l}21(38.9) \\
94(58)\end{array}$ & 0.018 \\
\hline $\begin{array}{l}\text { Hypertension } \\
\text { no }(n=17) \\
\text { yes }(n=199)\end{array}$ & $\begin{array}{c}0(0-37,992.5) \\
4,568(0-11,765)\end{array}$ & 0.477 & $\begin{array}{c}7(41.2) \\
108(54.3)\end{array}$ & 0.322 \\
\hline $\begin{array}{l}\text { Hyperuricemia } \\
\text { no }(n=121) \\
\text { yes }(n=71)\end{array}$ & $\begin{array}{c}14,620(386-2,0213) \\
0(0-9,707)\end{array}$ & 0.119 & $\begin{array}{l}72(59.5) \\
35(49.3)\end{array}$ & 0.179 \\
\hline $\begin{array}{l}\text { Hyperlipidemia } \\
\text { no }(n=24) \\
\text { yes }(n=192)\end{array}$ & $\begin{array}{c}0(0-1,646) \\
6,478(0-14,677)\end{array}$ & 0.046 & $\begin{array}{c}7(29.2) \\
108(56.2)\end{array}$ & 0.016 \\
\hline $\begin{array}{l}\text { NAFLD } \\
\text { no }(n=170) \\
\text { yes }(n=46)\end{array}$ & $\begin{array}{c}0(0-9,093) \\
14,580(1,049-29,852)\end{array}$ & 0.035 & $\begin{array}{l}84(49.4) \\
31(67.4)\end{array}$ & 0.032 \\
\hline $\begin{array}{l}\text { Metabolic syndrome } \\
\text { no }(n=75) \\
\text { yes }(n=141)\end{array}$ & $\begin{array}{c}0(0-2,686) \\
10,815(637-20,460)\end{array}$ & 0.002 & $\begin{array}{c}30(40) \\
85(60.3)\end{array}$ & 0.006 \\
\hline $\begin{array}{l}\text { Albuminuria } \\
\text { no }(n=98) \\
\text { yes }(n=118)\end{array}$ & $\begin{array}{c}0(0-7,678) \\
9,498(184-19,589)\end{array}$ & 0.117 & $\begin{array}{l}45(45.9) \\
70(59.3)\end{array}$ & 0.059 \\
\hline $\begin{array}{l}\text { Chronic kidney disease } \\
\text { no }(n=197) \\
\text { yes }(n=19)\end{array}$ & $\begin{array}{c}2,013(0-10,839) \\
18,993(0-48,799)\end{array}$ & 0.191 & $\begin{array}{c}103(52.3) \\
12(63.2)\end{array}$ & 0.472 \\
\hline
\end{tabular}

1 - significantly different from T2DM; ${ }^{2}$ - significantly different from controls; ${ }^{3}$ - significantly different form other groups; ${ }^{*}$ - assessed in person with diabetes, $\mathrm{n}=188$; ${ }^{* *}$ - assessed in clinical patients (without blood donors), $\mathrm{n}=216$; NAFLD - non-alcoholic fatty liver disease; ${ }^{2}$ - Mann-Whitney $U$ test; F - Fisher's exact test; $\uparrow$ - comparison of proportion between those 2 groups showed significant differences $(p=0.0025)$; bold - statistically significant results; $n$ - number of observations; AU - arbitrary units; T1DM - type 1 diabetes mellitus; T2DM - type 2 diabetes mellitus; T3DM - secondary type 2 diabetes mellitus; AGE-1 - advanced glycation end products; AGE-1 positivity - samples with measurable AGE-1 level (AGE-1 > 0).

The AGE-1-positive patients had higher concentrations of triglycerides $(153 \mathrm{mg} / \mathrm{dL}$ vs $140 \mathrm{mg} / \mathrm{dL}, \mathrm{p}=0.031)$, but not of total ( $p=0.666)$ or LDL cholesterol $(p=0.418)$, and lower concentrations of HDL cholesterol $(44.5 \mathrm{mg} / \mathrm{dL}$ vs $48 \mathrm{mg} / \mathrm{dL}, \mathrm{p}=0.020$ ).

Patients treated with lipid-lowering medications had significantly higher AGE-1 positivity than untreated patients (Table 3). However, the difference lost its significance when the analysis was restricted to treated against untreated patients diagnosed with hyperlipidemia (58\% vs $48.6 \%$, $\mathrm{p}=0.349)$.

\section{AGE-1 and hypertension, hyperuricemia and kidney function}

There was no significant difference in AGE-1 levels or AGE-1 positivity with respect to hypertension, hyperuricemia or the kidney function except for a tendency towards higher AGE-1 positivity in patients with albuminuria (Table 2). There was no correlation between AGE-1 and GFR index $(\rho=0.03, p=0.644)$.

Treatment with anti-hypertensive medications did not affect AGE-1 level or positivity (Table 3). 
Table 3. Effect of AGE-1 treatment on AGE-1

\begin{tabular}{|c|c|c|c|c|}
\hline Variables & $\begin{array}{l}\text { AGE-1 level }[\mathrm{AU}] \\
\text { median }(95 \% \mathrm{CI})\end{array}$ & $\mathrm{p}$-value ${ }^{\mathrm{M}}$ & $\begin{array}{c}\text { AGE-1 positivity } \\
n(\%)\end{array}$ & $p$-value ${ }^{F}$ \\
\hline $\begin{array}{l}\text { Antihypertensive drugs } \\
\text { no }(n=32) \\
\text { yes }(n=184)\end{array}$ & $\begin{array}{c}0(0-32,799) \\
4,888(0-12,313)\end{array}$ & 0.663 & $\begin{array}{c}14(43.7) \\
101(54.9)\end{array}$ & 0.256 \\
\hline $\begin{array}{l}\text { Dyslipidemia medications } \\
\text { no }(n=59) \\
\text { yes }(n=157)\end{array}$ & $\begin{array}{c}0(0-8,417) \\
6,814(104-16,832)\end{array}$ & 0.123 & $\begin{array}{l}24(40.7) \\
91(58)\end{array}$ & 0.032 \\
\hline $\begin{array}{l}\text { Aspirin } \\
\text { no }(n=75) \\
\text { yes }(n=141)\end{array}$ & $\begin{array}{c}0(0-6,643) \\
10,408(167-18,971)\end{array}$ & 0.020 & $\begin{array}{l}32(42.7) \\
83(58.9)\end{array}$ & 0.031 \\
\hline $\begin{array}{c}\text { Anticoagulants } \\
\text { no }(n=188) \\
\text { yes }(n=28)\end{array}$ & $\begin{array}{c}1,584(0-10,364) \\
19,289(0-32,988)\end{array}$ & 0.336 & $\begin{array}{l}98(52.1) \\
17(60.7)\end{array}$ & 0.424 \\
\hline $\begin{array}{l}\text { Clopidogrel } \\
\text { no }(n=188) \\
\text { yes }(n=28)\end{array}$ & $\begin{array}{c}4,068(0-14,447) \\
0(0-16,606)\end{array}$ & 0.486 & $\begin{array}{c}102(54.3) \\
13(46.4)\end{array}$ & 0.543 \\
\hline $\begin{array}{l}\text { Insulin } \\
\qquad \text { no }(n=130) \\
\text { yes }(n=86)\end{array}$ & $\begin{array}{l}4,069(0-15,947) \\
2,744(0-13,397)\end{array}$ & 0.574 & $\begin{array}{l}70(53.8) \\
45(52.3)\end{array}$ & 0.889 \\
\hline $\begin{array}{l}\text { Metformin } \\
\text { no }(n=74) \\
\text { yes }(n=142)\end{array}$ & $\begin{array}{l}1,584(0-10,894) \\
4,888(0-19,331)\end{array}$ & 0.164 & $\begin{array}{l}38(51.4) \\
77(54.2)\end{array}$ & 0.774 \\
\hline $\begin{array}{l}\text { Acarbose } \\
\text { no }(n=193) \\
\text { yes }(n=23)\end{array}$ & $\begin{array}{c}6,141(0-13,753) \\
0(0-13,207.5)\end{array}$ & 0.214 & $\begin{array}{l}107(55.4) \\
8(34.8)\end{array}$ & 0.077 \\
\hline $\begin{array}{l}\text { Sulfonylureas } \\
\text { no }(n=144) \\
\text { yes }(n=72)\end{array}$ & $\begin{array}{c}0(0-9,434) \\
12,069(44.5-24,259.5)\end{array}$ & 0.048 & $\begin{array}{l}71(49.3) \\
44(61.1)\end{array}$ & 0.113 \\
\hline $\begin{array}{l}\text { Gliptins } \\
\text { no }(n=184) \\
\text { yes }(n=32)\end{array}$ & $\begin{array}{c}263(0-9,015) \\
26,251(0-49,783)\end{array}$ & 0.016 & $\begin{array}{l}94(51.1) \\
21(65.6)\end{array}$ & 0.179 \\
\hline
\end{tabular}

M - Mann-Whitney U test; ${ }^{-}$- Fisher's exact test; bold - statistically significant results; AGE-1 - advanced glycation end products; AU - arbitrary units;

$n$ - number of observations; AGE-1 - advanced glycation end products.

\section{AGE-1 association with micro- and macroangiopathy and atherosclerosis}

Neither median AGE-1 level nor AGE-1 positivity differed significantly between patients with and without microangiopathy (Table 4). Also, there was no significant difference in AGE-1 level and AGE-1 positivity between patients with carotid arteries plaques and without them (Table 5).

Treatment with clopidogrel had no significant effect on AGE-1 level or positivity (Table 3).

\section{AGE-1 and inflammation}

The AGE-1-positive patients tended to have higher concentrations of hsCRP than patients with undetectable AGE-1 level $(2.58 \mathrm{mg} / \mathrm{dL}(2.0-3.3 \mathrm{mg} / \mathrm{dL})$ vs $2.07 \mathrm{mg} / \mathrm{dL}$ $(1.6-2.7 \mathrm{mg} / \mathrm{dL}), \mathrm{p}=0.227, \mathrm{n}=187)$. The difference in hsCRP between AGE-1-positive and -negative patients was significant when the analysis was limited to patients not treated with aspirin $(3.55 \mathrm{mg} / \mathrm{dL}(2.0-6.3 \mathrm{mg} / \mathrm{dL})$ vs $1.44 \mathrm{mg} / \mathrm{dL}$ $(0.98-2.10 \mathrm{mg} / \mathrm{dL}), \mathrm{p}=0.008)$. Also, there was a positive correlation between AGE-1 and hsCRP in these patients $(\rho=0.28, \mathrm{p}=0.029, \mathrm{n}=61)$.

Two-way ANOVA was applied to co-examine the effect of AGE-1 and aspirin treatment on hsCRP and showed that AGE-1 positivity $(\mathrm{p}=0.048)$ but not aspirin $(\mathrm{p}=0.687)$ was significantly associated with hsCRP.

Treatment with aspirin was associated with higher AGE-1 levels and frequency of AGE-1 positivity (Table 3).

\section{Multivariate analysis}

Logistic regression was applied to discern the independent predictors of AGE-1 positivity in patient cohort $(\mathrm{n}=216)$. All the variables significantly associated with AGE-1 positivity in univariate analysis, that is, hyperlipidemia, obesity (dichotomized), MetS, NAFLD, carotid artery plaques, and treatment with aspirin or lipid-lowering drugs, were entered into the analysis as explanatory variables. When analyzed exclusively in clinical patients, the effect of abnormal glucose metabolism did not reach statistical significance $(\mathrm{p}=0.088)$ and was not included. 
Table 4. AGE-1 association with microangiopathy

\begin{tabular}{|c|c|c|c|c|}
\hline Variables & $\begin{array}{l}\text { AGE-1 level [AU] } \\
\text { median }(95 \% \mathrm{CI})\end{array}$ & $\mathrm{p}$-value ${ }^{\mathrm{M}}$ & $\begin{array}{c}\text { AGE-1 positivity } \\
n(\%)\end{array}$ & $p$-value \\
\hline $\begin{array}{c}\text { Microangiopathy } \\
\text { no }(n=110) \\
\text { yes }(n=106)\end{array}$ & $\begin{array}{c}6,478(0-16,077.5) \\
1,584(0-12,172)\end{array}$ & 0.952 & $\begin{array}{l}59(53.6) \\
56(52.8)\end{array}$ & 1 \\
\hline $\begin{array}{l}\text { Retinopathy } \\
\qquad \begin{array}{c}\text { no }(n=186) \\
\text { yes }(n=30)\end{array}\end{array}$ & $\begin{array}{l}4,069(0-11,770) \\
2,316(0-41,540)\end{array}$ & 0.876 & $\begin{array}{l}99(53.2) \\
16(53.3)\end{array}$ & 1 \\
\hline $\begin{array}{c}\text { Nephropathy } \\
\text { no }(n=178) \\
\text { yes }(n=38)\end{array}$ & $\begin{array}{l}4,069(0-13,666) \\
1,584(0-19,253)\end{array}$ & 0.976 & $\begin{array}{l}94(52.8) \\
21(55.3)\end{array}$ & 0.859 \\
\hline $\begin{array}{c}\text { Polyneuropathy } \\
\text { no }(n=136) \\
\text { yes }(n=80)\end{array}$ & $\begin{array}{c}648(0-11,586) \\
5,533(0-19,643)\end{array}$ & 0.398 & $\begin{array}{l}69(50.7) \\
46(57.5)\end{array}$ & 0.397 \\
\hline
\end{tabular}

M - Mann-Whitney U test; ${ }^{F}$ - Fisher's exact test; AU - arbitrary units; $n$ - number of observation; AGE-1 - advanced glycation end products; AGE-1-positive - samples with measurable AGE-1 level (AGE-1 > 0).

Table 5. AGE-1 association with macroangiopathy

\begin{tabular}{|c|c|c|c|c|}
\hline Variables & $\begin{array}{l}\text { AGE-1 level [AU] } \\
\text { median }(95 \% \mathrm{CI})\end{array}$ & $p$-value ${ }^{\mathrm{M}}$ & $\begin{array}{c}\text { AGE-1 positivity } \\
n(\%)\end{array}$ & $p$-value ${ }^{F}$ \\
\hline $\begin{array}{l}\text { Macroangiopathy } \\
\text { no }(n=48) \\
\text { yes }(n=168)\end{array}$ & $\begin{array}{c}648(0-17,730) \\
4,069(0-12,309)\end{array}$ & 0.884 & $\begin{array}{l}25(52.1) \\
90(53.6)\end{array}$ & 0.871 \\
\hline $\begin{array}{l}\text { Ischemic heart disease } \\
\text { no }(n=136) \\
\text { yes }(n=80)\end{array}$ & $\begin{array}{l}2,316(0-12,393) \\
6,731(0-19,721)\end{array}$ & 0.723 & $\begin{array}{l}72(52.9) \\
43(53.7)\end{array}$ & 1 \\
\hline $\begin{array}{l}\text { Acute coronary syndromes } \\
\text { no }(n=171) \\
\text { yes }(n=45)\end{array}$ & $\begin{array}{l}1,155(0-11,516) \\
9,126(0-21,277)\end{array}$ & 0.685 & $\begin{array}{l}89(52) \\
26(57.8)\end{array}$ & 0.508 \\
\hline $\begin{array}{l}\text { Ischemic stroke } \\
\text { no }(n=191) \\
\text { yes }(n=25)\end{array}$ & $\begin{array}{c}1,155(0-11,299) \\
10,408(0-21,599)\end{array}$ & 0.728 & $\begin{array}{c}99(51.8) \\
16(64)\end{array}$ & 0.291 \\
\hline $\begin{array}{l}\text { Arteriosclerosis obliterans } \\
\text { no }(n=132) \\
\text { yes }(n=84)\end{array}$ & $\begin{array}{c}4,888(0-16,672) \\
0(0-13,080)\end{array}$ & 0.440 & $\begin{array}{l}74(56.1) \\
41(48.8)\end{array}$ & 0.329 \\
\hline $\begin{array}{l}\text { Carotid artery plaques } \\
\text { no }(n=73) \\
\text { yes }(n=143)\end{array}$ & $\begin{array}{c}0(0-3,984) \\
9,126(242-18,976)\end{array}$ & 0.053 & $\begin{array}{l}31(42.5) \\
84(58.7)\end{array}$ & 0.030 \\
\hline
\end{tabular}

M - Mann-Whitney U test; ${ }^{F}$ - Fisher's exact test; $95 \%$ CI - 95\% confidence interval; AU - arbitrary units; $n$ - number of observations; AGE-1 - advanced glycation end products; AGE-1-positive - samples with measurable AGE-1 level (AGE-1 > 0).

When co-examined, MetS $(\mathrm{p}=0.001)$, carotid artery plaques $(\mathrm{p}=0.006)$ and treatment with aspirin $(\mathrm{p}=0.018)$ contributed significantly to the prediction of AGE-1 positivity with the following ORs: $2.8(95 \% \mathrm{CI}=1.5-5.1), 2.4$ (95\% CI $=1.3-4.3)$ and $2.1(95 \% \mathrm{CI}=1.1-3.7)$, respectively. The effect of obesity, hyperlipidemia, NAFLD, and treatment with lipid-lowering drugs lost its significance.

To address the issue whether the effect of treatment with acarbose or gliptins on AGE-1 was independent from disparity in occurrence of metabolic abnormalities between treated and untreated patients, we repeated the analysis on a cohort of patients with abnormal glucose metabolism ( $n=198)$, introducing treatment with acarbose and gliptins as additional independent variables.

When co-examined, MetS $(\mathrm{p}=0.017)$, carotid artery plaques $(\mathrm{p}=0.007)$, NAFLD $(\mathrm{p}=0.030)$, and treatment with aspirin $(p=0.016)$ and acarbose $(p=0.008)$ contributed significantly to the prediction of AGE-1 positivity with the following ORs: 2.3 (95\% CI = 1.2-4.6), 2.5 (95\% CI = 1.3-4.8), 2.5 (95\% CI = 1.1-5.6), 2.2 (95\% CI = 1.2-4.1), and 0.25 (95\% CI $=0.09-0.7)$, respectively. The effect of obesity, hyperlipidemia and treatment with lipid-lowering drugs or gliptins lost its significance.

\section{Discussion}

Studies on albumin glycation have recently gained momentum with the focus of clinical research divided between the potential of glycated albumin as a biomarker and the effect of glycation on albumin affinity to drugs. ${ }^{20}$ Albumin glycation yields a plethora of diverse early and 
advanced products, frequently measured with methods using general properties of some of them, such as fluorescence, and referred to as "total AGEs". Analysis of specific products is less common and mostly limited to CML, CEL and the most abundant AGEs in human plasma ${ }^{21}$ - imidazolones - or their derivatives. ${ }^{20}$ To our knowledge, this is the first report on specific AGE-1 in patients with diabetes and related disorders, measured using commercially available monoclonal antibody directed against glucosemodified albumin, other than typically assessed glycation products. Similarly to other glucose-derived AGEs ${ }^{22}$ our AGE-1 was elevated in patients with diabetes as compared to healthy individuals; however, probably due to group size, the difference was significant exclusively for T2DM.

The formation of AGEs is heavily dependent on glucose concentration and reaction time; therefore, their accumulation is classically associated with diabetic complications. ${ }^{23-26}$ However, not all epidemiological studies have confirmed an independent association of AGEs with the incidence of diabetic nephropathy. ${ }^{27}$ Also, our AGE-1 was associated neither with polyneuropathy, nephropathy nor retinopathy. Still, AGE-1 accumulation was significantly increased in the presence of carotid artery plaques. Moreover, carotid plaques were found to be an independent predictor of AGE-1 positivity. Therefore, our observation corroborates the notion on other glucose-derived AGEs that they concentration is associated with the development of atherosclerosis in persons with diabetes..$^{22,28}$

Glycated albumin in all its forms is considered a promising biomarker for the prediction not only of diabetic complications but also of prediabetes. ${ }^{29}$ Accordingly, we demonstrated equally elevated AGE-1 levels, but not $\mathrm{HbA}_{1} \mathrm{C}$ levels, in prediabetes and diabetes. This seems to imply that the accumulation of our AGE- 1 is an earlier event than that of $\mathrm{HbA}_{1} \mathrm{c}$, despite the fact that formation of Amadori products, to which $\mathrm{HbA}_{1} \mathrm{c}$ belongs, precedes the synthesis of AGEs by months. It is possible that, in a manner similar to oxidative modification, albumin glycation may serve as a protective mechanism, slowing down the glycoxidation of more vital proteins. Corroborating our findings, Brunvand et al. ${ }^{30}$ reported that methylglyoxalderived hydroimidazolone-1, but not $\mathrm{HbA}_{1} \mathrm{c}$, was associated with early indications of diabetic cardiomyopathy. Yamagishi et al. ${ }^{31}$ found AGEs to contribute to the initial stages of diabetic nephropathy, while Heier et al. ${ }^{22}$ discovered that they redound to early steps of atherosclerosis. It should be mentioned, however, that pentosidine has been found to contribute to late events, such as coronary artery calcification. ${ }^{28}$

Diabetes is frequently accompanied by dyslipidemia, which contributes to micro- and macrovascular complications. Interestingly, we showed AGE-1 to be more tightly allied with abnormalities in lipid metabolism than hyperglycemia. With control group excluded, neither abnormal glucose metabolism was a predictor of AGE-1 positivity, nor AGE-1 correlated with glucose or $\mathrm{HbA}_{1} \mathrm{c}$.
In turn, AGE-1 was consistently associated with all lipid parameters. Moreover, MetS, encompassing 3 lipid-associated components, that is, reduced HDL cholesterol level, increased triglycerides and central obesity, was an independent predictor of AGE-1 positivity. This observation corroborates findings on CML and pentosidine, significantly associated with MetS or hyperlipidemia. ${ }^{23,32}$ Our results also agree well with a causative role attributed to AGEs in lipid accumulation. Yuan et al. ${ }^{26}$ showed that CML increased the expression of 3-hydroxy-3-methylglutaryl-coenzyme A (HMG-CoA) reductase, directly facilitating ectopic accumulation of lipids. Moreover, albumin modification alters its affinity towards fatty acids and translates into their increased blood concentration. ${ }^{20}$ Furthermore, a tight association between AGE-1 and lipid abnormalities is in line with findings showing that AGE inhibitors improve lipid profiles in persons with diabetes. ${ }^{33}$ Counterintuitively, treatment with lipid-lowering drugs in our cohort was associated with higher AGE-1 positivity. However, this might reflect the association of AGE-1 with dyslipidemia, since the effect was not observed in hyperlipidemic patients. Also, treatment with lipid-lowering drugs lost significance when co-analyzed with other variables.

Although not considered a diagnostic criterion for MetS, low-grade inflammation belongs to its satellite conditions. C-reactive protein, a marker of systemic inflammation, is elevated in MetS and increases gradually with the number of accumulated metabolic abnormalities. It is considered an independent predictor of diabetes and cardiovascular events that adds prognostic information to lipid screening. ${ }^{3}$ Low-grade inflammatory response and glycation are tightly intertwined. ${ }^{5,19}$ Indeed, with the antiinflammatory effect of aspirin accounted for, the positive association between glycation and low-grade inflammation could be observed in our cohort as well. Not only CRP levels were significantly higher in AGE-1-positive patients, but also AGE-1 directly correlated with CRP. Similarly, Heier et al. ${ }^{22}$ observed increased levels of methylglyoxal-derived hydroimidazolone-1 in the association with low-grade inflammation in T1DM.

Owing to its anti-inflammatory and anti-thrombotic activity, aspirin therapy is applied in primary and secondary prevention of cardiovascular events. ${ }^{34}$ Aspirin has been reported to inhibit glycation in vitro ${ }^{35}$ and lower tissue content of AGE, ${ }^{33}$ whereas its effect on circulating AGE has not been determined. Counterintuitively, there was a positive association between treatment with aspirin and AGE-1 in our cohort and aspirin treatment was a predictor of AGE-1 positivity, independent from MetS and plaque formation. Therefore, further mechanistic studies exploring aspirin effect on this particular AGE are warranted.

Formation of AGEs in people with diabetes is inhibited by rigorous glycemic control or metformin treatment. ${ }^{33}$ Accordingly, pentosidine, but not CML, has been significantly reduced in metformin-treated patients. ${ }^{32}$ However, 
AGE-1 in our cohort did not differ significantly with respect to the effectiveness of diabetes control and there was no correlation between AGE-1 and $\mathrm{HbA}_{1} \mathrm{c}$. Moreover, except for acarbose, any treatment intended to improve glucose metabolism was associated with higher AGE-1 levels, significantly so in the case of gliptins. Similarly, Haddad et al. ${ }^{32}$ found a strong tendency towards higher CML in MetS patients treated with metformin. In our cohort, this unexpected observation might be explained by the fact that treated patients had a significantly higher frequency of MetS, obesity or NAFLD. Indeed, when coexamined in multivariate analysis, the undesirable effect of gliptins on AGE-1 accumulation lost its significance. Nevertheless, altered drug effectiveness may contribute to the positive effect of treatment on AGE-1 accumulation. Albumin is a suitable transporter for a number of drugs and metabolites. ${ }^{36}$ However, its glycoxidation reduces its ability to prolong plasma circulatory time for low-molecular drugs and to assure their intracellular delivery. ${ }^{36}$ Moreover, any modification affects albumin-drug interaction, with the strength and the direction of the effect depending on the type and conditions of albumin modification as well as of the drug. ${ }^{20,36}$ Reduced availability of free, biologically active drug fraction may translate into improper control of glycemia ${ }^{20}$ and accelerate the formation of AGEs. Of note, fatty acids, frequently elevated in patients with metabolic abnormalities, have also been shown to affect the overall binding and affinity of native and glycated albumin towards sulfonylurea drugs. ${ }^{37}$

Unlike other evaluated glucose-lowering drugs, acarbose seems to be independently associated with reduced AGE-1 level. However, Tsunosue et al. ${ }^{38}$ reported that treatment with acarbose had no significant effect on postprandial plasma glucose. Instead, it decreased serum levels of glyceraldehyde-derived AGEs as well as those of free fatty acids. Acarbose has also been found to delay the progression of intima media thickening and reduce the incidence of cardiovascular disease. ${ }^{31}$ Observations made by our team and by Tsunosue et al. ${ }^{38}$ indicate that the beneficial effect of the drug might, at least partially, be mediated by AGE-lowering activity of acarbose.

Antibodies used in this study were directed against modified bovine and non-human serum albumin, which might be considered a limitation of our study. However, there is a great degree of homology between human and bovine albumin, and AGE-bovine albumin has been highly toxic when tested against human cell lines. ${ }^{31}$ Even more importantly, antibodies against mammal albumins are highly cross-reactive. ${ }^{39}$ The uncertainty as to which particular AGE epitopes are recognized by the antibodies used here is yet another limitation. The advantage of the method is, in turn, the monoclonal character of the antibodies. The producer claims no cross-reactivity towards the most typical AGEs, but there is no information concerning potential cross-reactivity towards others, e.g., pentosidine. However, taking into account that we did not find an association between our AGE-1 and diabetic microangiopathy or hypertension or that we have observed no metformin-associated reduction of AGE-1 accumulation reported for pentosidine in the literature, ${ }^{23,32}$ it is rather unlikely that antibodies used in our study recognize this particular AGE-1.

\section{Conclusions}

The dot-blot-slot method allows for the detection and quantification of non-standard AGE-1 epitopes, present in serum at low concentrations. The AGE-1, unlike typical AGEs, was more closely associated with the abnormalities in lipid than glucose metabolism. This finding confirms the diverse effects AGEs may have on the metabolism and stresses the need for their individual evaluation. Interestingly, only acarbose treatment was accompanied by decreased AGE-1 accumulation. The possible AGE-1-lowering effect of acarbose might be taken into account when planning treatment with antidiabetics to prevent lipidassociated complications.

This finding substantiates the notion on the diversity of the effects AGEs may have on the metabolism and stresses the need for their individual evaluation. Interestingly, only acarbose treatment was accompanied by decreased AGE-1 accumulation. The possible AGE-1-lowering effect of acarbose might be taken into account when planning treatment with antidiabetics to prevent lipid-associated complications.

\section{ORCID iDs}

Agnieszka Bronowicka-Szydełko

(1) https://orcid.org/0000-0001-9967-036X

Małgorzata Krzystek-Korpacka (10) https://orcid.org/0000-0002-2753-8092 Aleksandra Kuzan (D) https://orcid.org/0000-0003-4264-8174 Kinga Gostomska-Pampuch (D) https://orcid.org/0000-0002-0771-3893 Małgorzata Gacka (D) https://orcid.org/0000-0001-5760-1534 Urszula Jakobsche-Policht (1) https://orcid.org/0000-0002-5510-2675 Rajmund Adamiec (D) https://orcid.org/0000-0002-5616-5088 Andrzej Gamian (1) https://orcid.org/0000-0002-2206-6591

\section{References}

1. Pippitt K, Li M, Gurgle HE. Diabetes mellitus: Screening and diagnosis. Am Fam Physician. 2016;93(2):103-109.

2. World Health Organization. Global Reporton Diabetes. Geneva, Switzerland: World Health Organization; 2017.

3. Paoletti R, Bolego C, Poli A, Cignarella A. Metabolic syndrome, inflammation and atherosclerosis. Vasc Health RiskManag. 2006;2(2):145-152.

4. Sun YP, Gu JF, Tan XB, et al. Curcumin inhibits advanced glycation end product-induced oxidative stress and inflammatory responses in endothelial cell damage via trapping methylglyoxal. Mol Med Rep. 2016;13(2):1475-1486.

5. Nowotny K, Jung T, Höhn A, Weber D, Grune T. Advanced glycation end products and oxidative stress in type 2 diabetes mellitus. Biomolecules. 2015;5(1):194-222.

6. De Courten B, de Courten MP, Soldatos G, et al. Diet low in advanced glycation end products increases insulin sensitivity in healthy overweight individuals: A double-blind, randomized, crossover trial. Am J Clin Nutr. 2016;103(6):1426-1433.

7. Raghav A, Ahmad J, Alam K. Nonenzymatic glycosylation of human serum albumin and its effect on antibodies profile in patients with diabetes mellitus. PLoS One. 2017;12(5):e0176970. 
8. Indurthi VS, Leclerc E, Vetter SW. Interaction between glycated serum albumin and AGE-receptors depends on structural changes and the glycation reagent. Arch Biochem Biophys. 2012;528(2):185-196.

9. Takeuchi M. Serum levels of toxic AGEs (TAGE) may be a promising novel biomarker for the onset/progression of lifestyle-related diseases. Diagnostics (Basel). 2016;6(23):1-22.

10. Hohmann C, Liehr K, Henning C, et al. Detection of free advanced glycation end products in vivo during hemodialysis. J Agric Food Chem. 2017;65(4):930-937.

11. de Vos LC, Lefrandt JD, Dullaart RP, Zeebregts CJ, Smit AJ. Advanced glycation end products: An emerging biomarker for adverse outcome in patients with peripheral artery disease. Atherosclerosis. 2016; 254:291-299.

12. Ikeda $K$, Higashi $T$, Sano $H$, et al. $\mathrm{N}^{\epsilon}$-(carboxymethyl)lysine protein adduct is a major immunological epitope in proteins modified with advanced glycation end products of the Maillard reaction. Biochemistry. 1996;35(24):8075-8083.

13. Fu MX, Requena JR, Jenkins AJ, Lyons TJ, Baynes JW, Thorpe SR. The advanced glycation end product, $\mathrm{N}^{\epsilon}$-(carboxymethyl)lysine, is a product of both lipid peroxidation and glycoxidation reactions. J Biol Chem. 1996;271(17):9982-9986.

14. Takeuchi M, Makita Z, Yanagisawa K, Kameda Y, Koike T. Detection of noncarboxymethyllysine and carboxymethyllysine advanced glycation end products (AGE) in serum of diabetic patients. Mol Med. 1999;5(6):393-405.

15. World Health Organization. Obesity: Preventing and managing the global epidemic. Report of a World Health Organization Consultation. WHO Technical Report Series 894. Geneva, Switzerland: World Health Organization; 2000.

16. International Diabetes Federation. The IDF consensus worldwide definition of the metabolic syndrome. Brussels, Belgium: International Diabetes Federation; 2006. http://www.idf.org/metabolic-syndrome.

17. Catapano AL, Graham I, DeBackerG, Wiklund O,Chapman MJ, DrexelH. ESC/EAS Guidelines for the Management of Dyslipidemias. Eur Heart J. 2016;37(39):2999-3058.

18. Centers for Disease Control and Prevention. The Third National Health and Nutrition Examination Survey (NHANES III 1988-94) reference manuals and reports. Hyattsville, MD: National Center for Health Statistics; 1996

19. Yamagishi S, Inagaki Y, Okamoto T, Amano S, Koga K, Takeuchi M. Advanced glycation end product-induced apoptosis and overexpression of vascular endothelial growth factor and monocyte chemoattractant protein-1 in human-cultured mesangial cells. J Biol Chem. 2002;277(23):20309-20315.

20. Anguizola J, Matsuda R, Barnaby OS, et al. Review: Glycation of human serum albumin. Clin Chim Acta. 2013;425:64-76.

21. Thornalley PJ, Battah S, Ahmed N, et al. Quantitative screening of advanced glycation end products in cellular and extracellular proteins by tandem mass spectrometry. Biochem J. 2003;375(Pt 3):581-592.

22. Heier M, Margeirsdottir HD, Torjesen PA, Seljeflot I, Stensaeth KH, Gaarder M. The advanced glycation end product methylglyoxalderived hydroimidazolone-1 and early signs of atherosclerosis in childhood diabetes. Diab Vasc Dis Res. 2015;12(2):139-145.

23. Kerkeni M, Saïdi A, Bouzidi H, Letaief A, Ben Yahia S, Hammami M. Pentosidine as a biomarker for microvascular complications in type 2 diabetic patients. Diab Vasc Dis Res. 2013;10(3):239-245.
24. Marques CMS, Nunes EA, Lago L, et al. Generation of advanced glycation end-products (AGEs) by glycoxidation mediated by copper and ROS in a human serum albumin (HSA) model peptide: Reaction mechanism and damage in motor neuron cells. Mutat Res. 2017;824: 42-51.

25. Yu L, Zhang Y, Zhang H, Li Y. SOCS3 overexpression inhibits advanced glycation end product-induced EMT in proximal tubule epithelial cells. Exp Ther Med. 2017;13(6):3109-3115.

26. Yuan Y, Sun H, Sun Z. Advanced glycation end products (AGEs) increase renal lipid accumulation: A pathogenic factor of diabetic nephropathy (DN). Lipids Health Dis. 2017;16(1):126. doi:10.1186/s12944017-0522-6

27. Klein R, Horak K, Lee KE, Danforth L, Cruickshanks KJ, Tsai MY. The relationship of serum soluble receptor for advanced glycation end products (sRAGE) and carboxymethyl lysine (CML) to the incidence of diabetic nephropathy in persons with type 1 diabetes. Diabetes Care. 2017;40(9):117-119.

28. van Eupen MG, Schram MT, Colhoun HM, Scheijen JL, Stehouwer CD, Schalkwijk CG. Plasma levels of advanced glycation end products are associated with type 1 diabetes and coronary artery calcification. Cardiovasc Diabetol. 2013;12:149.

29. Bhat S, Jagadeeshaprasad MG, Venkatasubramani V, Kulkarni MJ. Abundance matters: Role of albumin in diabetes, a proteomics perspective. Expert Rev Proteomics. 2017;14(8):677-689.

30. Brunvand $L$, Heier $M$, Brunborg $C$, et al. Advanced glycation end products in children with type 1 diabetes and early reduced diastolic heart function. BMC Cardiovasc Disord. 2017;17(1):133. doi:10.1186/ s12872-017-0551-0

31. Yamagishi S, Matsui T, Ueda S, Fukami K, Okuda S. Clinical utility of acarbose, an alpha-glucosidase inhibitor in cardiometabolic disorders. Curr Drug Metab. 2009;10(2):159-163.

32. Haddad M, Knani I, Bouzidi H, Berriche O, Hammami M, Kerkeni M. Plasma levels of pentosidine, carboxymethyl-lysine, soluble receptor for advanced glycation end products, and metabolic syndrome: The metformin effect. Dis Markers. 2016;2016:6248264. doi:10.1155/ $2016 / 6248264$

33. Sourris KC, Harcourt BE, Forbes JM. A new perspective on therapeutic inhibition of advanced glycation in diabetic microvascular complications: Common downstream endpoints achieved through disparate therapeutic approaches? Am J Nephrol. 2009;30(4):323-335.

34. Ittaman SV, VanWormer JJ, Rezkalla SH. The role of aspirin in the prevention of cardiovascular disease. Clin Med Res. 2014;12(3-4):147-154.

35. Urios P, Grigorova-Borsos AM, Sternberg M. Aspirin inhibits the formation of pentosidine, a cross-linking advanced glycation end product, in collagen. Diabetes Res Clin Pract. 2007;77(2):337-340.

36. Larsen MT, Kuhlmann M, Hvam ML Howard KA. Albumin-based drug delivery: Harnessing nature to cure disease. Mol Cell Ther. 2016;4:3. doi:10.1186/s40591-016-0048-8

37. Basiaga SB, Hage DS. Chromatographic studies of changes in binding of sulfonylurea drugs to human serum albumin due to glycation and fatty acids. J Chromatogr B Analyt Technol Biomed Life Sci. 2010;878(30):3193-3197.

38. Tsunosue M, Mashiko N, Ohta Y, Matsuo Y, Ueda K, Ninomiya M. An alpha-glucosidase inhibitor, acarbose treatment decreases serum levels of glyceraldehyde-derived advanced glycation end products (AGEs) in patients with type 2 diabetes. ClinExpMed.2010;10(2):139-141.

39. Majorek KA, Porebski PJ, Dayal A, et al. Structural and immunologic characterization of bovine, horse and rabbit serum albumins. $\mathrm{Mol}$ Immunol. 2012;52(3-4):174-182. 